\title{
High-order harmonic generation in femtosecond laser micromachined devices
}

\author{
Anna G. Ciriolo ${ }^{1}$, Rebeca Martínez Vázquez ${ }^{1}$, Gabriele Crippa ${ }^{2}$, Davide Faccialà ${ }^{1}$, \\ Matteo Negro ${ }^{1}$, Michele Devetta ${ }^{1}$, Diogo Pereira Lopes ${ }^{2}$, Aditya Pusala ${ }^{2}$, Caterina Vozzi ${ }^{1}$, \\ Roberto Osellame ${ }^{1}$, and Salvatore Stagira ${ }^{1,2, *}$ \\ ${ }^{1}$ Istituto di Fotonica e Nanotecnologie IFN-CNR, piazza Leonardo da Vinci, 20133 Milano, Italy \\ ${ }^{2}$ Dipartimento di Fisica, Politecnico di Milano, piazza Leonardo da Vinci, 20133 Milano, Italy
}

\begin{abstract}
We demonstrate the generation of high-order harmonics in a fused-silica device fabricated through femtosecond laser micromachining. This achievement paves the way for the miniaturization of HHG applications from large-scale laboratories to microstructures.
\end{abstract}

\section{Introduction}

High-order harmonic generation (HHG) is nowadays largely exploited in Atomic and Molecular Physics, either as a spectroscopic probe or as a source of attosecond pulses for time-resolved measurements [1]. Beamlines based on HHG extend over several meters, since they operate in grazing incidence on bulky and expensive optics like toroidal mirrors and gratings. This instrumentation requires careful alignment and even active stabilization systems. Due to their technological complexity, HHG-based XUV-soft X coherent light sources are confined within a few number of advanced laboratories. In this framework, the miniaturization of HHG beamlines may produce a substantial breakthrough in ultrafast technology, with the potential for making HHG sources available for application in a number of different fields.

In this work, we demonstrate efficient $\mathrm{HHG}$ in a gas-filled microstructure fabricated through Femtosecond Laser Micromaching (FLM) followed by chemical etching in HF. Our goal was made possible by the advances in FLM techniques that are nowadays able to realize complex microchannels systems in monolithic devices with extreme flexibility, accuracy and 3D capabilities [2]. So far, these devices have been mainly applied to the transport of fluids, but they can also be used for manipulating gas fluxes and for guiding laser beams. Indeed, hollow waveguides (capillaries) are widely exploited in the field of ultrafast laser sources for the compression of intense pulses [3] and for the generation of high-order harmonics spectra up to the keV photon energy [4].

\section{Experimental method and results}

\footnotetext{
* Corresponding author: salvatore.stagira@polimi.it
} 
The microstructure we exploited is shown in Fig. 1(a); it consists of a fused silica plate (dimensions $6 \times 10 \times 1 \mathrm{~mm}$ ) containing two parallel microchannels; the upper one serves as auxiliary channel for beam alignment, whereas the lower one is devoted to HHG. The HHG channel has a $120-\mu \mathrm{m}$ diameter. A gas pipe was glued on a circular housing fabricated in the center of the chip, from which several smaller microchannels depart and carry the gas with uniform density along the main channel. HHG experiments were performed under vacuum conditions in a beamline composed of an interaction chamber and a grazing incidence XUV spectrometer. A fraction of a Ti:Sapphire laser output ( $25 \mathrm{fs}, 300 \mu \mathrm{J}, 1$ $\mathrm{kHz}$ ) was focused at the entrance of the microchannel located inside the interaction chamber. In order to optimize the coupling with the driving laser beam, the device was mounted on a high-precision motorized alignment stage. The gas density inside the channel was accurately tuned by means of a needle valve mounted within the gas line and the gas pressure was constantly monitored by means of a pressure gauge placed after the valve.
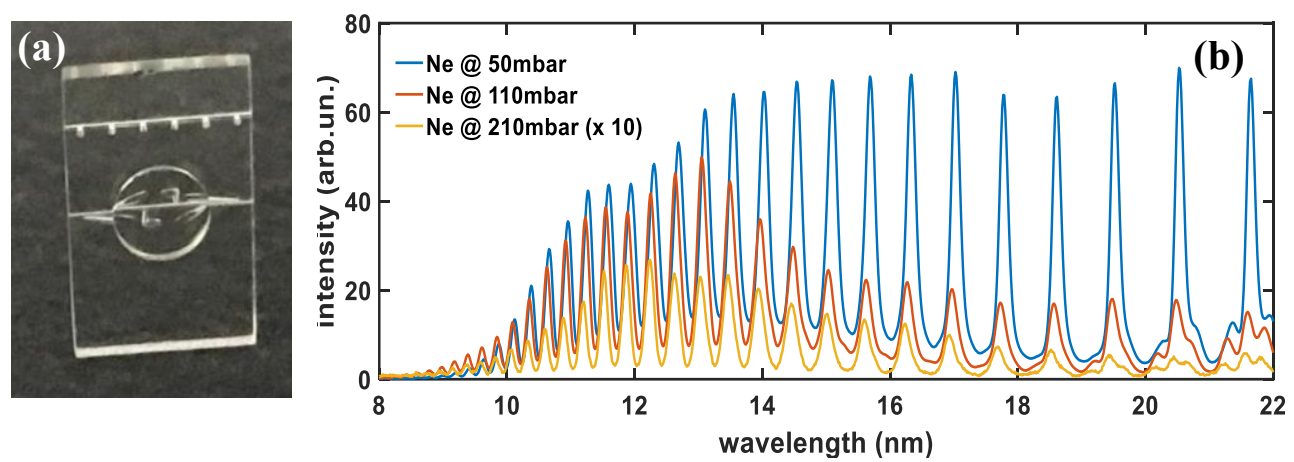

Figure 1 (a) Fused silica device endowed with a micro-channel fabricated by FLM. (b) HHG spectra generated inside the chip using Neon gas with a backing pressure of 50 (blue), 110 (red) and 210 mbar (yellow); this latter curve is magnified 10 times.

Figure 1(b) shows a comparison among different harmonic spectra generated inside the microchannel filled with neon gas. A decreasing yield is observed when the gas backing pressure is increased from 50 to 210 mbar. The reduction is more evident for low-order harmonics, as indicated by the reshaping of the harmonic spectrum. This pressure dependence is due to both phase-matching effects and absorption from the gas: as the pressure increases, the XUV radiation is strongly absorbed; moreover, the phase mismatch between the fundamental and the harmonic field worsens and, owing to the long interaction region where the process occurs, it leads to a dramatic reduction of the yield mainly in the low-energy part of the spectrum.

We compared the HHG yield inside the microstructure with that achieved in the most commonly used interaction geometry based on a gas jet. Figure 2(a) shows single-shot harmonics spectra generated inside the channel with helium at a 300-mbar backing pressure (blue line); the results are compared to HHG in a steady jet with 1-mm diameter at same gas pressure (red line, x1000 magnification) and in a pulsed jet with same diameter at a 4bar backing pressure (yellow line, x5 magnification). An extended cutoff, up to $7 \mathrm{~nm}$ (160 $\mathrm{eV})$ is obtained inside the microchannel, whereas a cutoff of about $11 \mathrm{~nm}(110 \mathrm{eV})$ is observed in the jets. Moreover, a higher generation yield is achieved in the microchannel, up to 20 times the pulsed jet and more than $10^{4}$ times the steady one.

We speculate that the improved performances achieved in the microchannel are related both to the extension of the interaction region and to the different interaction geometry with 
respect to the gas jets. We also expect that a further improvement in the performances of the channel-based scheme can be obtained by overcoming the phase-matching limitations. In this sense, we are currently investigating a different device. In particular, we are studying HHG from a microchannel endowed with multiple gas nozzles (see picture 2(b)). Due to the nozzles arrangement, a periodic modulation of the gas density distribution will be obtained, such as to allow for HHG in a Quasi-Phase Matching (QPM) regime. The gas flow inside the channel and the harmonics generation process upon QPM will be also theoretically explored with the support of advanced microfluidic simulations and HHG propagation simulations.



Figure 2 (a) Comparison between HHG spectra produced in helium inside the microchannel (blue), in a continuous gas jet (red) and in a pulsed gas jet (yellow). (b) QPM device. It is composed of a main channel with a periodic modulation of the diameter, multiple gas nozzles and small channels for gas

\section{Conclusions}

In conclusion, we have demonstrated the generation of high order harmonics from a gasfilled structured microchannel realized through Femtosecond Laser Micromachining. This approach outperforms the standard gas jets-based configuration both in terms of yield and cutoff extension.

We foresee that the large flexibility of FLM may give access to novel HHG sources where the yield and the cutoff extension could be further improved by properly shaping the microchannel and by properly engineering the gas density distribution. On a longer term, the potentialities of FLM might lead to the realization of miniaturized HHG beamlines, where several functionalities (attosecond pulse generation, pump-probe measurements, spectral acquisition etc.) could be integrated inside the same platform without the use of grazing incidence optics.

\section{References}

1. F. Krausz and M. Ivanov, Rev. Mod. Phys. 81, 163 (2009).

2. R. R. Gattass and E. Mazur, Nature Photonics 2, 219 (2008).

3. M. Nisoli et al, IEEE JSTQE 4, 414 (1998)

4. T. Popmintchev et al., Science 336, 1287 (2012) 